\title{
Parkinson's disease: an open label trial of pergolide in patients failing bromocriptine therapy
}

\author{
STEWART A FACTOR, JUAN R SANCHEZ-RAMOS, WILLIAM J WEINER \\ From the University of Miami School of Medicine, Department of Neurology, Miami, Florida, USA
}

SUMMARY Sixty-three patients with Parkinson's disease who failed bromocriptine therapy for various reasons were treated in an open-label trial of pergolide. The data were evaluated in a retrospective manner. Forty-six percent had a good response and tolerated the pergolide. A comparison of the outcomes regarding response and toxicity revealed that bromocriptine and pergolide act differently in individual patients. A trial of pergolide in Parkinsonian patients failing bromocriptine therapy may be therapeutically useful.

The efficacy of pergolide mesylate in Parkinson's disease has been well established. Both double blind ${ }^{1-4}$ and open label trials ${ }^{4-7}$ have demonstrated improvement in almost all features of Parkinson's disease as well as the clinical fluctuations in response associated with long term levodopa therapy. Pergolide, like bromocriptine, is an ergot derivative with dopamine receptor agonist properties. Despite the fact that pergolide differs from bromocriptine pharmacologically ${ }^{89}$ and structurally, ${ }^{910}$ clinical trials comparing the two have revealed similar responses and toxicity in individual patients. ${ }^{9}$ We evaluated the efficacy of pergolide in Parkinsonian patients failing bromocriptine therapy in an effort to assess three issues. First, can pergolide be beneficial in those patients with Parkinson's disease who fail bromocriptine therapy because of lack of efficacy, loss of efficacy, or adverse effects? Second, does a similar pattern of response or lack of response occur in individual patients? Third, are the adverse effects which a patient experiences while treated with bromocriptine predictive for the presence and type of adverse effects observed during pergolide therapy?

\section{Patients and methods}

Patients Sixty three patients, 31 male and 32 female, with Parkinson's disease who had previously failed bromocriptine therapy were enrolled in an open label trial of pergolide. The results were evaluated in a retrospective manner. The average patient age was 74 (ranging from 44 to 80 ) and the duration of disease averaged 12.5 years (ranging from 2 to 27). Hoehn and Yahr stages ranged from 2 to 5 .

Address for reprint requests: William J Weiner, MD, University of Miami School of Medicine, Department of Neurology (D4-5), PO Box 016960 , Miami, Florida 33101.

Received 18 August 1987 and in revised form 9 November 1987. Accepted 17 November 1987
All patients signed informed consent documents. In order to qualify for entry into the pergolide program, symptoms of Parkinson's disease had to be inadequately controlled with levodopa/carbidopa (Sinemet) and bromocriptine. Inadequate control was defined as an absence of improvement or continued decline in the patients' ability to perform the activities of daily living or a continued worsening of the signs and symptoms of Parkinson's disease. In addition, patients experiencing intolerable adverse reactions from bromocriptine were considered failures. Although not all patients were initially started on bromocriptine by us, all patients were examined by at least one of the authors at the maximum dosage reached and again after withdrawal from the medication before pergolide therapy was initiated. An adequate trial of bromocriptine required that a minimum dosage of $25 \mathrm{mg}$ per day be reached or, in those patients not tolerating doses of this level, a minimum of two trials be attempted. Women of childbearing age and patients with serious pulmonary, hepatic, renal or cardiac diseases were excluded.

Medication Pergolide mesylate was available as $50 \mathrm{mcg}$, $0.1 \mathrm{mg}, 0.25 \mathrm{mg}$, and $1.0 \mathrm{mg}$ capsules for the first 24 months of the trial and then 0.25 and $1.0 \mathrm{mg}$ tablets for the last 10 months. Patients entering the study during the first 24 months followed a dosage schedule which started with 50 mcg per day with breakfast. This dosage was maintained for 2 days and then increased to $50 \mathrm{mcg}$ bd with meals for the next 2 days. If this dosage was well tolerated the medication was then increased by $100 \mathrm{mcg}$ per day until a daily dosage of $0.8 \mathrm{mg}$ was reached. Thereafter the dose was titrated to an optimum therapeutic level by increments of 0.1 to $0.2 \mathrm{mg}$ not more frequently than alternating days. After day 4, pergoilide was given twice or thrice daily with meals and a bedtime dose was given if necessary. Patients entering the study during the last 10 months started at a dosage of 0.125 $\mathrm{mg}$ per day (a half of a $0.25 \mathrm{mg}$ tablet) with breakfast. The patients were maintained on this dosage for 4 days and then increased by increments of $0.125 \mathrm{mg}$ not more frequently than alternating days until an optimum dosage was reached. We observed no difference between the 2 dosage schedules in the ability to tolerate the pergolide. The total daily dosage 
ranged from 0.25 to $6 \mathrm{mg}$ per day and the duration of therapy ranged from 1 to 34 months. Levodopa/carbidopa was continued when patients were entered into the study and the daily dosage ranged from $37 \cdot 5 / 150 \mathrm{mg}$ to $150 / 1500 \mathrm{mg}$. The dosage of levodopa/carbidopa was gradually reduced as therapeutic levels of pergolide were reached to avoid overmedication. These reductions were made slowly to avoid any loss of efficacy. Bromocriptine was discontinued at least 14 days prior to entry. However, patients receiving other concomitant therapy (anticholinergics and amantidine) were allowed to continue on these medications.

Study design Patients were evaluated at 2 week intervals for the first month while the pergolide was titrated. Thereafter, patients were seen at 3 month intervals. Pre-therapy examination included a complete history (including detailed history of therapeutic trials with bromocriptine regarding dosage, duration and adverse effects) and physical examination. Patients were also required to have a chest radiograph, electrocardiogram, urinalysis, complete blood count with differential and platelet count, and a full serum chemistry profile. During therapy patients were required to have a urinalysis, complete blood count with differential and platelet count, and serum chemistry profile every visit. Patients were questioned in detail as to the effect of the medication on activities of daily living including use of kitchen utensils, hygiene, turning in bed, gait and balance, and tremor. In addition, the patients were questioned about the symptoms of Parkinson's disease including leg dystonia, freezing and fluctuations. All information was recorded in a standard history. Examination at each visit included supine and standing blood pressure and neurological examination to assess the Parkinson's disease. Tremor, rigidity, bradykinesia, gait difficulties (including problems with balance and posture), clinical fluctuations, dyskinesia, freezing, leg dystonia, and voice difficulties were all assessed during the examination. The Hoehn and Yahr scale ${ }^{11}$ was utilised for staging the Parkinson's disease. All adverse effects were monitored.

Comparison of bromocriptine and pergolide Patient records were reviewed regarding the outcome of bromocriptine therapy and its effects on activities of daily living and the signs and symptoms of Parkinson's disease. In addition, reasons for withdrawal were assessed and included lack of efficacy, loss of efficacy, or adverse effects. Lack of efficacy was defined as a lack of improvement and a continued decline in ability to perform activities of daily living and in the signs and symptoms of Parkinson's disease. Loss of efficacy involved a return to pre-treatment status despite escalations in drug dosage after previous improvement was noted in activities of daily living and signs and symptoms of Parkinson's disease. Patients withdrawing from bromocriptine therapy were then placed into one to four categories regarding pattern of response or lack of response. In category one six patients suffered from loss of efficacy; category two 19 received $25 \mathrm{mg}$ or more of bromocriptine with a complete lack of efficacy; category three four received $25 \mathrm{mg}$ or more of bromocriptine with a lack of efficacy plus adverse effects at the higher dosage; and category four 34 patients had intolerable adverse effects at dosages lower than $25 \mathrm{mg}$. Using the same outcome categories (a dosage of $4 \mathrm{mg}$ or greater being considered an adequate trial) each patient was then evaluated regarding their response to pergolide. The treatments were then compared for each patient in an attempt to deter- mine if the outcome of bromocriptine therapy was similar to the outcome of pergolide therapy. A comparison of the adverse effects of bromocriptine and pergolide was also performed to ascertain whether or not patients suffered from the same side effects with both drugs. The question as to whether the presence of adverse effects from bromocriptine were predictive for the presence of the same effects from pergolide was evaluated. A reverse comparison was also performed to examine whether or not adverse effects of pergolide were predictive for the same effects from bromocriptine.

\section{Results}

Efficacy Of the 63 patients who failed bromocriptine therapy $29(46 \%)$ had a positive result with pergolide. A positive (good) result was defined as an improvement in the performance of activities of daily living as described by the patient and observed by the examiner and improvement of the signs and symptoms of Parkinson's disease as documented by the examiner. The effects of pergolide on individual features of Parkinson's disease is summarised in table 1. The Hoehn and Yahr stage improved in $16(25 \%)$ of the patients, remained unchanged in $42(67 \%)$, and worsened in five $(8 \%)$. Sinemet dose was decreased in $29(46 \%)$ of the patients, remained unchanged in 24 $(38 \%)$, and was increased in $10(16 \%)$. Good responses have been maintained for over a year in 14 patients with six of these patients continuing to do well after 2 years. The longest duration of a positive response has been 34 months. Three patients died for reasons unrelated to drug therapy. Twelve patients had an early good response followed by a partial or complete loss of efficacy. Seven of these patients continue to be better than baseline and have continued to take pergolide while three have returned to baseline and have withdrawn from the study.

Comparison of the outcome of bromocriptine and pergolide therapy The result of the administration of

Table 1 Efficacy of pergolide on the signs and symptoms of Parkinson's disease

\begin{tabular}{|c|c|c|c|c|}
\hline $\begin{array}{l}\text { Signs and } \\
\text { symptoms }\end{array}$ & $\begin{array}{l}\text { Total } \\
\text { patients }\end{array}$ & $\begin{array}{l}\% \\
\text { improved }\end{array}$ & $\begin{array}{l}\% \\
\text { unchanged }\end{array}$ & $\begin{array}{l}\% \\
\text { worsened }\end{array}$ \\
\hline $\begin{aligned} 1 & \text { Tremor } \\
2 & \text { Rigidity } \\
3 & \text { Bradykinesia } \\
4 & \text { Gait } \\
5 & \text { Freezing } \\
6 & \text { Wearing-off } \\
7 & \text { On-Off } \\
8 & \text { Dyskinesia } \\
9 & \text { Leg Dystonia } \\
10 & \text { Voice }\end{aligned}$ & $\begin{array}{l}29 \\
36 \\
40 \\
54 \\
29 \\
18 \\
23 \\
29 \\
17 \\
11\end{array}$ & $\begin{array}{r}65 \cdot 5 \\
52 \cdot 8 \\
47 \cdot 5 \\
50 \cdot 0 \\
6 \cdot 9 \\
38 \cdot 9 \\
52 \cdot 2 \\
27 \cdot 6 \\
52 \cdot 9 \\
72 \cdot 7\end{array}$ & $\begin{array}{l}31 \cdot 0 \\
44 \cdot 4 \\
50 \cdot 0 \\
44 \cdot 4 \\
69 \cdot 0 \\
44 \cdot 4 \\
39 \cdot 1 \\
31 \cdot 0 \\
47 \cdot 1 \\
27 \cdot 3\end{array}$ & $\begin{array}{r}3 \cdot 5 \\
2 \cdot 8 \\
2 \cdot 5 \\
5 \cdot 6 \\
24 \cdot 1 \\
16 \cdot 7 \\
8 \cdot 7 \\
41 \cdot 4 \\
0 \cdot 0 \\
0 \cdot 0\end{array}$ \\
\hline
\end{tabular}

The total patients column refers to the number of patients in which the sign or symptom listed on the left was moderate or severe and bothersome. The right 3 columns refer to the percent of those patients who improved, remained unchanged or worsened. Pergolide was efficacious in $46 \%$ of the patients. It was helpful in all aspects of Parkinson's disease with the exception of freezing. 
either pergolide or bromocriptine was not the same. Of the patients in category one of bromocriptine response four have withdrawn from pergolide; three because of lack of efficacy and one because of the presence of intolerable adverse effects. Of the two patients maintained on pergolide, one continues to have a good response while the other has had some loss of efficacy. Of the patients in category two, 10 have stopped pergolide, five because of lack of efficacy, four because of intolerable adverse effects and one because of death unrelated to drug therapy. Nine other patients in this category continue to take pergolide; three continue to do well, one has had a loss of efficacy, two have had a lack of efficacy, and three others have had a lack of efficacy with adverse effects at doses of $4 \mathrm{mg}$ or greater. Of the patients in category three, two have withdrawn from pergolide therapy because of lack of efficacy and two have continued with one having a good response and the other suffering from some loss of efficacy. Finally, of the patients in category four, 12 have withdrawn from pergolide, one for loss of efficacy, four for lack of efficacy, four because of adverse effects with doses less than $4 \mathrm{mg}$, one with lack of efficacy and adverse effects at dosages of $4 \mathrm{mg}$ or greater and two died. Twenty two of these patients have continued pergolide with 15 continuing to have a good response, five with no response, and one with adverse effects at low doses, and one with a loss of efficacy.

Adverse effects Forty-one of the 63 patients had adverse effects of some kind when treated with pergolide. The number of patients and the different adverse effects from both pergolide and bromocriptine are listed in table 2. Psychiatric effects were the most common and included hallucinations, confusion and one

Table 2 Adverse effects of pergolide and bromocriptine and the number of patients experiencing them

\begin{tabular}{lll}
\hline & $\begin{array}{l}\text { Number of patients } \\
\text { on pergolide with } \\
\text { adverse effects }(\%)\end{array}$ & $\begin{array}{l}\text { Number of patients } \\
\text { on bromocriptine with } \\
\text { adverse effects }(\%)\end{array}$ \\
Adverse effect & $21(33 \cdot 3)$ & $22(34.9)$ \\
\hline Psychiatric & $6(9 \cdot 5)$ & $10(15 \cdot 9)$ \\
Dyskinesia & $6(9 \cdot 5)$ & $3(4 \cdot 8)$ \\
Nausea/vomiting & $5(7 \cdot 9)$ & $0(0)$ \\
Urinary difficulties & $5(7 \cdot 9)$ & $8(12 \cdot 7)$ \\
Postural faintness & $3(4 \cdot 8)$ & $4(6 \cdot 4)$ \\
Anxiety & $3(4 \cdot 8)$ & $3(4 \cdot 8)$ \\
Fatigue & $2(3 \cdot 2)$ & $2(3 \cdot 2)$ \\
Headache & $1(1 \cdot 6)$ & $0(0)$ \\
Acute dystonia & $1(1 \cdot 6)$ & $1(1 \cdot 6)$ \\
Hair loss & $1(1 \cdot 6)$ & $0(0)$ \\
Increased appetite & $1(1 \cdot 6)$ & $0(0)$ \\
Recurrence of angina & $1(1 \cdot 6)$ & $0(0)$ \\
Tingling sensations & $0(0)$ & $1(1 \cdot 6)$ \\
Rash & $0(0)$ &
\end{tabular}

Forty-one patients had adverse effects of some variety but in only 11 were they intolerable requiring withdrawal from the study. Thirty eight patients had adverse effects from bromocriptine, 34 of which were severe enough to require withdrawal of therapy. patient who complained of mental slowing. In only 11 of the 41 patients were the adverse effects intolerable requiring withdrawal of the medication. Seven had hallucinations. Of the other four patients, withdrawal from therapy was because of recurrence of angina pectoris, gastritis, urinary difficulties and the occurrence of tingling sensations. Adjustments of the pergolide and/or the levodopa/carbidopa dosage usually alleviated the adverse effects in the other patients.

Thirty-eight of the 63 patients had experienced adverse effects with bromocriptine. In 34 they were severe enough to warrant withdrawal from therapy. These side effects were similar to those of pergolide. A comparison of the side effects in individual patients of bromocriptine and pergolide revealed that patients generally did not suffer from the same side effects with both medications (table 3). A reverse comparison revealed that adverse effects from pergolide were not predictive for those from bromocriptine (table 4).

Table 3 Comparison of the adverse effects of bromocriptine and pergolide

\begin{tabular}{lrl}
\hline Adverse effects & $\boldsymbol{A}$ & $\boldsymbol{B}$ \\
\hline Psychiatric & 22 & 12 \\
Dyskinesia & 10 & 1 \\
Postural faintness & 8 & 0 \\
Anxiety & 4 & 1 \\
Nausea/vomiting & 3 & 2 \\
Fatigue & 3 & 0 \\
Rash & 2 & 0 \\
Headache & 2 & 1 \\
Hair loss & 1 & 1 \\
Nightmares & 1 & 0 \\
\hline
\end{tabular}

Column A refers to the number of patients experiencing the side effects listed on the left with bromocriptine. Column B refers to the number of patients in column A experiencing the same adverse effects with pergolide. Adverse effects with bromocriptine were not predictive for the same difficulties with pergolide in individual patients.

Table 4 Reverse comparison of the adverse effects of bromocriptine and pergolide

\begin{tabular}{lrr}
\hline Adverse effects & $A$ & $B$ \\
\hline Psychiatric & 21 & 12 \\
Dyskinesia & 6 & 1 \\
Nausea/vomiting & 6 & 2 \\
Urinary difficulties & 5 & 0 \\
Postural faintness & 5 & 0 \\
Anxiety & 3 & 1 \\
Fatigue & 3 & 0 \\
Headache & 2 & 1 \\
Hair loss & 1 & 1 \\
Acute dystonia & 1 & 0 \\
Increased appetite & 1 & 0 \\
Recurrence of angina & 1 & 0 \\
Tingling sensations & 1 & 0 \\
\hline
\end{tabular}

Column A refers to the number of patients experiencing the side effects listed on the left with pergolide. Column B refers to the number of patients in column A experiencing the same adverse effects with bromocriptine. Adverse effects with pergolide were not predictive for the same difficulties with bromocriptine in individual patients. 
In addition to the clinical effects some patients had laboratory abnormalities. Eight patients $(12 \cdot 7 \%)$ had a transient anaemia, five $(7.9 \%)$ had mild elevation of liver enzymes, three $(4.8 \%)$ had mild reversible elevations in serum creatinine, one had a transient elevation in blood urea nitrogen levels and one had hypoglycaemia which may not have been drug related.

\section{Discussion}

Levodopa/carbidopa continues to be the treatment of choice in Parkinson's disease. The long term side effects, including loss of efficacy and motor fluctuations in response to the medication, unfortunately, limit its usefulness. These difficulties have served as the impetus to the study of dopamine agonists as a secondary line of treatment in Parkinson's disease. Bromocriptine, the most widely used agonist, is not efficacious in all Parkinson's disease patients with loss of efficacy and fluctuations in response to levodopa/carbidopa. In addition, in our experience, one cannot predict which patient will and will not do well with bromocriptine. One goal of therapeutic trials of other dopamine agonists is to discover an agent which will be efficacious in those patients with a poor response to levodopa and bromocriptine. In a comparison of bromocriptine and pergolide in 24 patients by LeWitt $e t a l^{9}$ a similar degree of efficacy and the same adverse effects were experienced by individual patients. It would seem from this information that an individual's response to bromocriptine would predict a similar response to pergolide. However, in a later report on 11 patients, Goetz et $a l^{12}$ found that pergolide could be effective in patients with a loss of efficacy to bromocriptine. Leiberman et al ${ }^{10}$ examined 25 patients and observed that pergolide was useful in patients with loss of efficacy and intolerable side effects from bromocriptine but did not assess the response of individual patients to both medications. In our study we retrospectively evaluated the efficacy of pergolide in patients with a poor response to bromocriptine as manifested by a loss of efficacy, lack of efficacy or intolerable side effects. After evaluation of individual response to the two drugs we conclude that pergolide can be efficacious in almost one half of Parkinson's disease patients who are bromocriptine failures. The outcome of bromocriptine therapy does not predict a similar outcome for pergolide therapy. Finally, individual patients do not necessarily experience the same adverse effects from both drugs and may easily tolerate one drug while poorly tolerating the other. Pergolide and bromocriptine appear to act differently in individual patients. Pergolide would be a useful addition to the currently available spectrum of therapeutic agents for Parkinson's disease. Further prospective studies would be useful in further substan- tiating these observations.

The efficacy of pergolide in this study was similar to previous observations of its use in Parkinson's disease patients. However, we note that pergolide was useful in controlling foot and leg dystonia associated with Parkinson's disease. Although the same results were not encountered by Tanner et al, ${ }^{7}$ dopamine agonists have previously been utilised with some success in this situation. ${ }^{13} \mathrm{~A}$ trial of pergolide in patients with Parkinson's disease and associated leg dystonia is certainly reasonable in those patients not responding to baclofen. ${ }^{14}$ Pergolide was also observed to be generally ineffective in the treatment of freezing episodes. This finding is consistent with previous reports. ${ }^{46}$

This retrospective study of the use of pergolide in Parkinson's disease patients who have failed a trial of bromocriptine presents evidence that therapeutic outcome to dopamine agonists in Parkinson's disease is not determined by a single dopamine agonist trial. Individual patient response to different agonists cannot be predicted at this time and successive agonist trials with the potential to achieve better therapeutic results are indicated in the management of Parkinson's disease.

This work was supported by NIH grant \# NS07238 and by the National Parkinson Foundation. Pergolideo was supplied by Eli Lilly and Company. This paper was presented in part at the 39th annual Americano Academy of Neurology meeting April 1987, New? York.

\section{References}

1 Diamond SG, Markham CH, Treciokas LJ. Doubleblind trial of pergolide for Parkinson's disease. Neurology 1985;35:291-5.

2 Jankovic J, Orman J. Parallel double-blind study of pergolide in Parkinson's disease. In: Yahr MD, Bergmann KJ, eds. Advances in Neurology 1986:551-4.

3 Olanow CW, Alberts MJ. Double-blind controlled study of pergolide mesylate as an adjunct to Sinemet in the treatment of Parkinson's disease. In: Yahr MD, Bergmann KJ, eds. Advances in Neurology 1986: 555-60.

4 Lang AE, Quinn N, Brincat S, Marsden CD, Parkes JD. Pergolide in late-stage Parkinson's disease. Ann Neurol 1982;12:243-7.

5 Tanner CM, Goetz CG, Glantz RH, Glatt SL, Klawans HL. Pergolide mesylate and idiopathic Parkinson's disease. Neurology 1982;32:1175-9.

6 Jankovic J. Long-term study of pergolide in Parkinson's disease. Neurology 1985;35:296-9.

7 Tanner CM, Goetz CG, Glantz RH, Klawans HL. Pergolide mesylate: four years' experience in Parkinson's disease. In: Yahr MD, Bergmann KJ, eds. Advances in Neurology 1986;547-9. 
8 Koller WC, Weiner WJ, Diamond BI, Nausieda PA, Klawans HL. The pharmacological evaluation of pergolide mesylate as a potential anti-parkinson agent. Neuropharmacol 1980;19:831-7.

9 LeWitt PA, Ward CD, Larsen TA, et al. Comparison of pergolide and bromocriptine therapy in Parkinsonism. Neurology 1983;33:1009-14.

10 Leiberman AN, Neophytides A, Leibowitz M, et al. Comparative efficacy of pergolide and bromocriptine in patients with advanced Parkinson's disease. In: Fahn S, Calne DB, Shoulson I, eds. Advances in Neurology 1983;95-108.
11 Hoehn MM, Yahr MD. Parkinsonism: onset, progression, and mortality. Neurology 1967;17: 427-42.

12 Goetz CG, Tanner CM, Glantz RH, Klawans HL. Chronic agonist therapy for Parkinson's disease: a 5-year study of bromocriptine and pergolide. Neurology 1985;35:749-51.

13 Quinn NP, Koller WC, Lang AE, Marsden CD. Painful Parkinson's disease. Lancet 1986;i:1366-9.

14 Nausieda PA, Weiner WJ, Klawans HL. Dystonic foot response of Parkinsonism. Arch Neurol 1980;37:132-6.

\section{The Ramsay Hunt syndrome}

"This syndrome may be divided into three clinical groups: (1) Herpes zoster auricularis, (2) Herpes zoster in any of the zoster zones of the cephalic extremity (herpes auricularis, herpes facialis, and herpes occipito-collaris) with facial palsy, (3) Herpes zoster of the cephalic extremity with facial palsy and auditory symptoms (tinnitus, deafness, vertigo, vomiting, nystagmus and disturbances of equilibrium)".

Hunt J Ramsay. On herpetic inflammations of the geniculate ganglion. A new syndrome and its complications. J Nerv Ment Dis 1907; 34:73-96.

\section{Todd's paralysis}

JB Lyons" states "Todd's right to the eponym 'Todd's Paralysis' is indisputable. Postictal paralysis is described in the Lumleian Lectures for $1849^{2}$ : "A paralytic state remains sometimes after the epileptic convulsion. This is more particularly the case when the convulsion has affected only one side or one limb: that limb or limbs will remain paralytic for some hours, or even days, after the cessation of the paroxysm, but it will ultimately perfectly recover-".

\section{References}

RT ROsS 1 Lyons JB. The Neurology of Robert Bentley Todd in Historical Aspects of the Neurosciences. In: Rose FC, Bynum WF, eds. New York: Raven Press, 1981:145.

2 Todd RB. London Medical Gazette 1849;8:668 